\title{
Annie Jourdan (dir.), Louis Bonaparte. Roi de Hollande
}

\section{Cyril Triolaire}

\section{(2) OpenEdition \\ 1 Journals}

\section{Édition électronique}

URL : https://journals.openedition.org/ahrf/11916

DOI : 10.4000/ahrf.11916

ISSN : 1952-403X

Éditeur :

Armand Colin, Société des études robespierristes

\section{Édition imprimée}

Date de publication : 1 décembre 2010

Pagination : 198-201

ISBN : 978-2-200-92634-2

ISSN : 0003-4436

\section{Référence électronique}

Cyril Triolaire, «Annie Jourdan (dir.), Louis Bonaparte. Roi de Hollande », Annales historiques de la Révolution française [En ligne], 362 | octobre-décembre 2010, mis en ligne le 25 mars 2011, consulté le 23 avril 2022. URL : http://journals.openedition.org/ahrf/11916; DOl : https://doi.org/10.4000/ahrf. 11916

Ce document a été généré automatiquement le 23 avril 2022.

Tous droits réservés 


\title{
Annie Jourdan (dir.), Louis Bonaparte. Roi de Hollande
}

\author{
Cyril Triolaire
}

\section{RÉFÉRENCE}

Annie Jourdan (dir.), Louis Bonaparte. Roi de Hollande, Paris, Nouveau Monde éditions/ Fondation Napoléon, 2010, 511 p., ISBN 978-2-84736-492-7, $26 €$

1 À la suite de la lecture du livre dirigé par Annie Jourdan, professeur associé à l'Université d'Amsterdam, l'image trop répandue et longtemps entretenue par l'historiographie classique d'un Louis Bonaparte mélancolique, hypocondriaque, maladroit, docile, faible et médiocre, s'évanouit. En réunissant très exclusivement à ses côtés la fine fleur universitaire néerlandaise spécialiste de la révolution batave et des années napoléoniennes - dix-neuf archivistes, doctorants, enseignants-chercheurs, historiens, historiens des arts, du droit et de la pensée politique -, Annie Jourdan présente le jeune frère de l'empereur sous un nouveau visage. Au fil des communications, Louis Bonaparte apparaît en homme mûr, autoritaire, sûr de lui, de ses choix et de sa politique. C'est un "bon roi», apprécié de ses sujets et dont il respecte les opinions, un monarque d'inspiration française ayant accepté de se faire Hollandais, un prince soucieux de "nationaliser sa monarchie et de devenir un roi national ». Car là réside tout l'intérêt de ce portrait peint à plusieurs mains. Non seulement le caractère, les motivations, les ambitions et les devoirs du roi Louis Bonaparte sont finement saisis mais en plus, la mise en place d'une monarchie inédite dans un pays républicain et de tradition fédéraliste ainsi que le dilemme de son attachement à la France et de sa fidélité envers sa nouvelle patrie sont mis en lumière de manière très juste. Si l'agencement final laisse penser que l'ouvrage aurait pu être mieux chapitré - les vingt communications auraient mérité d'être réparties en plusieurs grandes parties, qui s'imposent par ailleurs de fait -, leur diversité autant, généralement, que leur qualité en font un ouvrage majeur. Publié avec le soutien des fondations Daendels et Napoléon, cet opus propose la reproduction de près de cent 
gravures, peintures, cartes, plans et photographies ; souvent réduite à la taille d'un timbre et exclusivement en noir et blanc, l'iconographie présentée n'en demeure pas moins riche, ce qui fait d'autant plus regretter au lecteur les économies réalisées par l'éditeur sur la taille et la couleur des illustrations - certaines, piètres, auraient même finalement mérité de « disparaître » (p. 219, 266, 365, 432).

2 Annie Jourdan ouvre cet ouvrage collectif en rappelant la genèse du royaume néerlandais et le processus qui conduit Louis, l'étranger, le catholique, au trône de la très républicaine Hollande. S'il devient prince par la seule volonté de son frère, si le traité de Paris fixe bien les contours d'un régime monarchique constitutionnel, reconnaissant à la fois les principes de la révolution batave et ses symboles, c'est lui, l'ex-commandant de l'armée de réserve, qui définit une majesté toute "nationale ", capable de souder toute la communauté néerlandaise. Il est un homme de son temps, l'héritier d'un despotisme éclairé, le dépositaire d'une certaine rationalisation et uniformisation de l'État hollandais. Le propos liminaire de l'historienne pose ainsi très clairement et les enjeux de la formation du nouvel État, et les moyens engagés par Louis pour y parvenir, dans un contexte très délicat - gestion de la conscription dans un pays allergique à l'esprit militaire, conciliation délicate des restrictions imposées par le blocus et de la protection de son économie, concurrence des egos familiaux à l'heure de l'affirmation d'une nouvelle nation. Si tous les efforts consentis par Louis sont loin d'être couronnés de succès, s'il n'arrive jamais complètement à s'imposer comme un Hollandais à part entière et à être pleinement adopté par sa communauté nationale, il se projette en revanche toujours et tente autant de structurer que de moderniser son nouvel état. C'est cette "nationalisation de sa monarchie " qu'Annie Jourdan et ses collaborateurs décryptent en prêtant toute leur attention aux initiatives et aux grands chantiers embrassés entre le printemps 1806 et l'été 1810 , dans un tableau stimulant.

3 La formation d'un État national est au cœur des développements des six premiers chapitres. En se penchant sur la mort de la république, Wyger Velema s'intéresse à l'établissement même de la monarchie et éclaire un épisode délaissé par l'historiographie. En rappelant qu'existent alors de réels débats sur la nature du régime le plus approprié à la Hollande, il insiste sur la nécessité de relire les premières heures du nouveau royaume et souligne combien sa proclamation résulte alors autant d'un affaiblissement conceptuel du républicanisme que du renoncement des Néerlandais. À l'intelligente politique menée par Louis - défense des intérêts néerlandais, engagement vers une réconciliation nationale et centralisation administrative -, Renger de Bruin ajoute également les effets de l'essoufflement de la démocratie représentative, après treize ans d'expériences plurielles et un enthousiasme progressivement douché par les coups d'État de 1798 et 1801 - l'arrivée au pouvoir du frère de l'empereur portant seulement le coup de grâce à une influence populaire déclinante. Structurer l'État passe alors par un assainissement et une modernisation du système des finances publiques, une codification du droit et une réorganisation du pouvoir judiciaire. Wantje Fritschy réhabilite pleinement le rôle joué par Louis pour asseoir la fiscalité et contrôler au mieux les comptes du royaume et ce, malgré des divergences entre le souverain et Gogel. Car l'unification est au cœur de la politique de Louis, qu'elle soit budgétaire ou législative; c'est d'elle que dépend principalement l'élaboration rapide d'un «État national ». Peter Van den Berg et Maarten Van Boven le montrent respectivement de façon pertinente : la définition d'un droit propre, indigène, le processus de codification 
et l'uniformisation de l'organisation judiciaire procèdent d'une même formation d'État et d'une même affirmation identitaire. L'émergence d'un droit " hollandais national ", rompant avec les particularismes locaux, et d'une justice inédite, parfois d'inspiration française, relèvent d'une seule démarche unificatrice. Le prince Louis mesure les dangers représentés par l'inertie régnant dans son nouveau royaume et la défiance des républicains les plus acharnés; Joke Roelevink rappelle que la transposition d'un modèle français n'est pas suffisante à la construction d'un État hollandais uni et que le roi Bonaparte trace une voie nationale inédite.

L'établissement du royaume de Hollande s'insérant dans le cadre d'une lutte économique et militaire contre l'Angleterre, Johan Joor se demande si son affirmation est finalement compatible avec la stratégie du blocus imposée par l'empereur. S'il a bien essayé de satisfaire aux exigences de son frère - réglementation, création d'organes de surveillance - pour en tempérer un moment les foudres, Louis échoue, la corruption et la fraude facilitées par la géographie le desservant autant que ses choix assumés en faveur de l'activité des marchands de sa nouvelle nation. Bien qu'il eût été préparé à une simple vie de militaire, rappelée trop linéairement par Jos Gabriëls, et qu'il fut cantonné à l'arrière de la scène aux premières heures de l'empire, le roi Louis, Grand Dignitaire et Connétable, remplacé et humilié aux avant-postes guerriers, tente au contraire de maintenir fermement la barre dans son royaume, au risque d'irriter à Paris. Aussi, ce désir de souder autour de lui la communauté néerlandaise ne s'exprime pas seulement à travers certains de ses choix économiques, fermement contestés ailleurs, il passe également par la création d'un nouvel ordre de chevalerie empreint de tradition néerlandaise et l'adoption d'un système moderne de décorations d'inspiration française, sur lesquels revient habilement Georges Sanders. Louis souhaite que le processus d'unification du royaume se fasse autour de lui. L'élaboration et l'affirmation de sa nouvelle identité royale passe de fait par un art pensé de la représentation; au point de nourrir une contre-image - recourant aux figures traditionnelles du tyran et du despote hier utilisées contre le stadhouder et aujourd'hui contre l'empereur -, bientôt à l'origine du façonnement d'une "identité néerlandaise post-napoléonienne " d'après Matthijs Lok, et même de servir de « modèle » au nouveau prince Guillaume, en pleine Restauration, selon une idée supposée «nouvellement reçue » et que relisent de concert Ido de Haan et Jeroen Van Zanten. Sa majesté nationale, Louis l'exprime à travers son vif désir de connaître, de modeler et de s'approprier son royaume. Et les six dernières communications de l'ouvrage en rendent parfaitement compte.

Sa rationalisation projetée du nouvel État néerlandais passe par une politique sociétale aussi originale que dynamique, et dont l'analyse dévoile combien la volonté de Louis fut grande et ses initiatives finalement nombreuses et variées. Aussi voit-il très tôt dans l'instruction publique un moyen pour favoriser l'unité nationale et former des citoyens hollandais vertueux. Martijn Van der Burg montre ainsi que si le roi Louis hérite d'un système scolaire profondément renouvelé par la révolution batave, c'est lui qui prolonge la réforme et fait entrer en vigueur la législation. Soucieux de moderniser l'université, de promouvoir l'enseignement secondaire et professionnel, il multiplie les efforts pour soutenir l'école primaire et ce, même si sa sécularisation est peu réussie et ses ambitions de nationalisation et d'uniformisation confrontées à diverses résistances. D'autres grands chantiers sont ouverts ou activés par un prince Lodewijk souhaitant être apprécié de tous les Hollandais. L'intérêt qu'il leur manifeste et qu'il prête à leur artisanat, à leur industrie, à leur bien être, à leur environnement et à leurs arts renvoie à l'accomplissement de son projet pour une monarchie nouvelle selon Eveline Koolhass- 
Grosfeld. Toute la société néerlandaise devrait pouvoir profiter de cet élan. La véritable "politique culturelle nationale » à laquelle il œuvre, pour reprendre les mots d'Aagje Gosliga, concourt à la définition et à la promotion d'un art d'intention patriote; cette politique à succès se traduit par des commandes nationales. Le style français n'est pas seulement copié, il est réapproprié, dépassé, stimulant et développant les arts décoratifs, les beaux arts et un artisanat de luxe "hollandais». La politique centralisatrice conduite par Louis dans les domaines des arts et des sciences favorise incontestablement les arts plastiques contemporains « néerlandais » comme le souligne pertinemment Ellinoor Bergvelt. La fondation même d'un Musée Royal des Beaux Arts renvoie à sa double envie d'ouverture d'un lieu de formation d'excellence pour les artistes et de création de collections permanentes publiques des grands maîtres hollandais vivants. Malgré une politique d'achats manquant de cohérence et lacunaire, Louis jette indéniablement les bases d'une vie culturelle hollandaise moderne. Son rapport personnel et public à l'architecture témoigne aussi de ce choix d'une politique réellement nationale; Thomas Von der Dunk la met parfaitement en lumière dans un chapitre aussi copieux que stimulant et offre un regard neuf sur cette période francobatave délaissée par les historiens des arts depuis Ozinga. L'espace remodelé serait moins prestigieux s'il n'avait été par ailleurs assaini, au nom du bien être de toute une nation. Esther Starkenburg montre ainsi comment ce roi de constitution fragile prit un soin particulier à fixer des règles d'hygiène devant garantir la santé de chacun, pour celle de tous. C'est alors tout un programme de transformation de la ville, esthétique et sanitaire, et de promotion d'une bonne circulation de l'air, d'un accès à l'eau potable, d'une alimentation saine et d'exercices physiques quotidiens, que Louis initie, mais que son frère ne lui laisse finalement pas le temps de mettre en œuvre. Bien que satellite de l'empire français et bientôt annexé, le royaume de Hollande commence à renouveler son identité grâce à la politique du prince Louis.

6 Pour Annie Jourdan, « le roi [Louis] a compris qu'une monarchie tempérée, dotée d'une constitution unitaire et d'un souverain modéré remplacerait avantageusement une république oligarchique, déchirée par des siècles de querelles partisanes » (p. 417). Tout le mérite des auteurs de ce livre est d'avoir su relire ce court épisode franco-batave et de saisir comment, en cherchant à définir une voie nationale et à devenir un roi national, Louis façonne une identité et un nouvel esprit hollandais, en mariant avec habileté son inspiration française et l'héritage néerlandais. Les fausses notes observées sur la forme - découpage des chapitres, qualité de l'iconographie - et le fond - topiques éculés mais rappelés sans distance sur le caractère du roi $(p .113,145)$, mise en garde critique à relativiser contre une nouvelle idée reçue supposée (p. 213-216), vocabulaire hésitant («monarchie pseudo-absolue », p. 276) - sont au final suffisamment rares pour ne retenir de cet ouvrage collectif qu'il est avant tout une synthèse riche et incontournable sur le roi Louis Bonaparte. 